\title{
N9 $0-22991$
}

Aspects of Efficient and Reliable Multibody System Simulation

R. Schwertassek and C. Führer

Institut für Dynamik der Flugsysteme

and

W. Rulka

MAN Technologie

\begin{abstract}
Multibody system equations can be generated in various forms. All of these may be interpreted as results of two basic approaches, the augmentation-and the elimination-method. The former method yields the descriptor for $m$ of the system motion, a set of differential-algebraic equations (DAE), and the latter the state space representation, a minimal set of ordinary differential equations (ODE). Both of these methods are surveyed. Particular emphasis is on the discussion of recursive computational schemes, generating the equations of motion with a number of operations, which is proportional to the number $N$ of system bodies $(O(N)$-for mulations).

For simulation purposes one would like to create that set of system equations, which can be generated most efficiently and for which the most efficient and reliable solution techniques are available. Numerical solution techniques for ODE have been studied in great detail and they are welldeveloped. By contrast, DAE have not been investigated for such a long time. In view of new developments in the latter field the generation of all the equations required for an efficient and reliable solution of DAE describing multibody system motion is discussed. These methods, i.e. an $O(N)$-formulation and new techniques for solving DAE, are implemented in the SIMPACK code. Its capabilities are illustrated by simulation of multibody robot models.
\end{abstract}

